\title{
Cross sections for production of closed superstrings at high energy colliders in brane world models
}

\author{
Diego Chialva, ${ }^{1,2}$ Roberto Iengo, ${ }^{1,2}$ and Jorge G. Russo ${ }^{3}$ \\ ${ }^{1}$ International School for Advanced Studies (SISSA), Via Beirut 2-4, I-34013 Trieste, Italy \\ ${ }^{2}$ INFN, Sezione di Trieste, Trieste, Italy \\ ${ }^{3}$ Institució Catalana de Recerca i Estudis Avançats (ICREA), Departament ECM, Facultat de Física, Universitat de Barcelona, \\ Barcelona, Spain
}

(Received 23 March 2005; published 27 May 2005)

\begin{abstract}
In brane world string models with large extra dimensions, there are processes where fermion and antifermion (or two gluons) can annihilate producing a light particle (e.g. gluon) carrying transverse momentum and a Kaluza-Klein graviton or an excited closed string that propagates in the extra dimensions. In high energy colliders, this process gives a missing-momentum signature. We compute the total cross section for this process within the context of type II superstring theory in the presence of a D-brane. This includes all missing-energy sources for this string-theory model up to $s=8 M_{s}^{2}$, and it can be used to put new limits on the string scale $M_{s}$.
\end{abstract}

DOI: 10.1103/PhysRevD.71.106009

PACS numbers: 11.25.Mj, 04.50.+h, 12.90.+b

\section{INTRODUCTION}

The existence of D-branes has opened the possibility that our Universe may have large (as compared to a fundamental or string length) extra dimensions where only gravity propagates [1]. Because the gravitational force is weak, the size of extra dimensions might be as large as $O(0.1) \mathrm{mm}$ [2], for two large extra dimensions, and $O\left(10^{-11}\right) \mathrm{mm}$, for six large extra dimensions. Large extra dimensions imply that gravitational forces can become strong already at $1-10 \mathrm{TeV}$. This leads to the exciting prospective that quantum gravity effects could be seen at LHC.

String theory provides a theoretical framework to study the possible quantum gravity physics at the $\mathrm{TeV}$ scale. An interesting process is the production of closed-string states, gravitons but also other massless and massive states, that will propagate in the extra dimensions and will appear as missing energy in the experiments [3].

Studies of such processes where the closed string is a Kaluza-Klein graviton appeared in the literature [4-7] (other effects of Kaluza-Klein particles were investigated in [8-11]). Here we will focus on processes with quarks and gluons in the initial states and an arbitrary (missing) closed-string state and a gluon or a quark in the final state.

We will follow the approach of [7]: in the tendimensional spacetime there are $N$ coincident D3-branes stretched out in three uncompact dimensions (our observed world), and the remaining six dimensions are compactified on a torus with periodicity $\left(2 \pi R_{m}\right), m=1, \ldots, 6$. The massless states associated with open strings that end on the D3-brane are described by $\mathcal{N}=4$ super Yang-Mills (YM) theory with gauge group $\mathrm{U}(N)$ and the massless spectrum of open-string states is thus given by the $\mathcal{N}=$ 4 supermultiplet. Following [7] we reinterpret its vectors and spinors as gluon and quarks.

The present model [7] is oversimplified, but it is already capable of illustrating in a quantitative way the pattern that one should expect to measure in a high energy collider due to missing-energy processes, when the center of mass (CM) energy overcomes the string scale. We have made accurate calculations, taking care of factors 2 and $\pi$, even though we are aware that this is not a precision test of string theory.

We study the scattering of two gluons or of a quark and an antiquark or of a gluon and a quark giving as a final state a (missing) massless or massive closed string and a gluon or a quark having a sizable transverse momentum with respect to the incident beam. Hopefully the final state will experimentally appear as a detectable violation of transverse momentum conservation.

We consider the limit of transverse momentum $p_{T}$ small as compared to the string momentum scale $M_{s}=1 / \sqrt{\alpha^{\prime}}$ [but, given that $M_{s}>O(1) \mathrm{TeV}, p_{T}$ could be large as, say, $0.05 \mathrm{TeV}$ or even more]. In this limit we obtain a very simple expression for the cross section, in the form of the stringy cross section for producing the closed-string state from the initial state, as a function of the total CM energy only, times a simple factor containing the dependence on $p_{T}$ and the emission angle $\theta$. In a sense our case resembles a bremsstrahlung process where the "soft" emitted particle is the final gluon (or quark).

The string-theory cross section for producing the closedstring state from an initial state made of two open strings can be obtained exactly from the imaginary part of the forward scattering of two open strings at one nonplanar loop. This scattering amplitude is known in closed form in ten dimensions $[12,13]$, and it can be easily generalized to the case of open strings on a Dp-brane [7]. The cross section obtained in this way holds for any energy and encodes all the allowed Neveu-Schwarz Neveu-Schwarz (NS-NS) and Ramond-Ramond (R-R) (ten-dimensional) massless and massive closed-string states, incorporating also the Kaluza-Klein (KK) contribution. This method is particularly useful in string theory, given the rapid growth 
of the number of states at higher masses (for example, in type II theory for level number $N=1$ there are already some thousands of propagating degrees of freedom).

Of course, in computing the imaginary part we do not meet any of the divergences, which instead can occur in the real part (the divergences in the real part led to some puzzles in the evaluation of diagrams with virtual intermediate KK closed-string states $[6,7,9])$.

One distinctive feature of string theory, as opposed to other attempts to incorporate gravity in the theory, is the occurrence of an infinite number of unstable excited states, which appear as resonances in the cross section.

In the present case the resonances are due to open-string intermediate states of mass $\alpha^{\prime} M^{2}=N$, with odd $N$. These are essentially the poles that appear in scattering amplitudes in D-branes involving two open and one closed string found by [14]. This characterizing signature of string theory could be seen in missing transverse momentum processes in colliders - and at the parton level it is particularly clean - by looking at the dependence of the cross section on $s$ (the total CM energy squared) either at fixed $p_{T}$ and $\theta$, or also after integrating over those variables in their allowed range.

We present our results for $0 \leq \alpha^{\prime} s \leq 8$, corresponding to the production of closed-string states with (tendimensional) mass $\alpha^{\prime} M^{2}=4 N$ and $N=0,1$.

One crucial ingredient to make quantitative estimates is the resonance widths that regularize the poles. They are typically given by $\alpha_{s} M_{s}$ times some numerical coefficient, where $\alpha_{s}$ is the strong coupling constant, $\alpha_{s}=g_{\mathrm{YM}}^{2} / 4 \pi$. We have estimated the widths of the first resonances for various channels (using the results of Ref. [7]) and used them as indicative average widths of the higher ones. ${ }^{1} \mathrm{We}$ find two typical widths: $3.4 \alpha_{s} M_{s}$ and $7.4 \alpha_{s} M_{s}$ for gluongluon and quark-antiquark initial states respectively; the comparison of the results for the two cases can give an idea on the dependence on the widths.
Furthermore, some splitting of the resonance multiplets will occur, due, in particular, to supersymmetry breaking. This will lead to a splitting of some peaks in the cross section (this splitting will eventually be smoothed out when including the effect of the parton distributions).

Plots of the parton level cross sections for $g g \rightarrow g+$ missing and $q \bar{q} \rightarrow g+$ missing are shown in Sec. V. We find parton level cross sections of order $25 \mathrm{pb} \times$ $\left(1 \mathrm{TeV}^{2} / M_{s}^{2}\right)$ and $7 \mathrm{pb} \times\left(1 \mathrm{TeV}^{2} / M_{s}^{2}\right)$ respectively, for the first peak.

\section{TOTAL CROSS SECTION FOR OPEN + OPEN TO CLOSED STRING}

The particles in a high energy collider (electron-positron or quarks and gluons) are represented in the string model by massless open-string states. In this section we are interested in the total cross section for two open massless string states going into an arbitrary closed-string state, in the presence of a D3-brane. In the absence of extra emission of a visible particle, this process is not observed in colliders, since the closed-string state escapes to other dimensions and it cannot be detected. However, we consider this process in detail since it will illustrate the method and some formulas will be used for the calculation of the missing-momentum process of Sec. IV.

Let us consider, for the beginning, the case of a $\mathrm{D}_{p}$-brane in general. Take as $p_{1}^{\mu}, p_{2}^{\nu}, \mu, \nu=0,1, \ldots, 3$ the momentum of the ingoing open strings and $P^{\hat{\mu}}, \hat{\mu}=0,1, \ldots, 9$, the momentum of the outgoing closed string. The center of mass energy is $s=2 p_{1} \cdot p_{2}$, with metric $\eta_{\mu \nu}=$ $\operatorname{diag}(1,-1,-1,-1)$. In the presence of the brane, translational invariance is broken in $9-p$ transverse directions and there is only conservation of momentum along the $p$ directions of the brane. We choose the center of mass frame where $P_{\hat{\mu}}=\left(P_{0}, \overrightarrow{0}, P_{m}\right), m=p+1, \ldots, 9$. In general, the formula for the cross section is as follows:

$$
\begin{aligned}
\sigma_{2 \rightarrow 1}(s) & =\frac{1}{2 s} \int \frac{d^{9} P}{2(2 \pi)^{9} P_{0}}\left|A_{2 \rightarrow 1}\right|^{2}(2 \pi)^{p} \delta^{(p)}\left(\vec{p}_{1}+\vec{p}_{2}-\vec{P}\right)(2 \pi) \delta\left(E_{1}+E_{2}-\sqrt{M^{2}+\vec{P}^{2}}\right) \\
& =\frac{1}{2 s} \int \frac{d^{9-p} P}{2(2 \pi)^{8-p} P_{0}}\left|A_{2 \rightarrow 1}\right|^{2} \delta\left(E_{1}+E_{2}-\sqrt{M^{2}+\vec{P}^{2}}\right) \\
& =\operatorname{Vol}\left(\Omega_{8-p}\right) \frac{1}{4 s}(2 \pi)^{p-8}\left|A_{2 \rightarrow 1}(s)\right|^{2}\left(s-M^{2}\right)^{(7-p) / 2}, \\
\operatorname{Vol}\left(\Omega_{8-p}\right) & =\frac{2 \pi^{(9-p) / 2}}{\Gamma\left(\frac{9-p}{2}\right)} .
\end{aligned}
$$

Remarkably, the cross section does not have a peak at $s=$ $M^{2}$. On the contrary, it vanishes. The reason is that now $s=M^{2}$ is a threshold for the production of the state which

\footnotetext{
${ }^{1}$ For a calculation of widths of closed-string states, see [15].
}

in general will have $P_{m}$ transverse momentum components, and right on the threshold $s=M^{2}$ the phase space vanishes.

The total cross section for the production of an arbitrary closed-string state from two open-string states can be obtained directly by taking the imaginary part of the am- 
plitude corresponding to the nonplanar annulus diagram for forward scattering of $2 \rightarrow 2$ open strings (with two vertex operators on the outer boundary, and two vertex operators on the inner boundary). This amplitude was obtained long ago by Green and Schwarz [12,13]. In the presence of a Dp-brane, we modify it by inserting, in the integrand over the modulus $\tau$, the factor $(\pi \tau)^{(p-9) / 2}$ to take into account the nonconservation of the momentum transverse to the Dp-brane (see the analysis below and also [7]):

$$
\begin{aligned}
A_{D p}= & \mathcal{N}_{p} K 2^{-2 \alpha^{\prime} s} \pi \int d \tau(\pi \tau)^{(p-9) / 2} e^{(\pi \tau / 2) \alpha^{\prime} s} \\
& \times \int d \nu_{12}\left(\sin \pi \nu_{12}\right)^{-\alpha^{\prime} s} \\
& \times \int d \nu_{13}\left(\sin \pi \nu_{13}\right)^{-\alpha^{\prime} s} G\left(q, \nu_{i}\right)
\end{aligned}
$$

where

$$
G\left(q, \nu_{i}\right)=\prod_{n=1}^{\infty} \frac{\left(1-2 \cos \left(2 \pi \nu_{13}\right) q^{2 n-1}+q^{4 n-2}\right)^{\alpha^{\prime} s}\left(1-2 \cos \left(2 \pi \nu_{24}\right) q^{2 n-1}+q^{4 n-2}\right)^{\alpha^{\prime} s}}{\left(1-2 \cos \left(2 \pi \nu_{12}\right) q^{2 n}+q^{4 n}\right)^{\alpha^{\prime} s}\left(1-2 \cos \left(2 \pi \nu_{13}\right) q^{2 n}+q^{4 n}\right)^{\alpha^{\prime} s}}, \quad q=e^{-\pi \tau} .
$$

Setting $\xi_{1}=\xi_{4}, \xi_{2}=\xi_{3}, t=0, s=-u$ (forward scattering), the kinematical factor for the case of two gluons reduces to (see [13])

$$
K=\left(\alpha^{\prime} s\right)^{2} \xi_{2} \cdot \xi_{3} \xi_{1} \cdot \xi_{4}=\left(\alpha^{\prime} s\right)^{2} \xi_{2}^{2} \xi_{1}^{2},
$$

while for the case of two fermions (in the forward case $1 \rightarrow$ $4,2 \rightarrow 3$ )

$$
K=2\left(\alpha^{\prime} s\right) \alpha^{\prime} \bar{u}_{1} \gamma^{\mu} u_{1} \bar{u}_{2} \gamma_{\mu} u_{2} .
$$

As we will take into account color quantum numbers later on, here we omit Chan-Paton factors and, for a D3-brane, we take the same normalization constant $\mathcal{N}_{3}$ as in [7]

$$
\mathcal{N}_{3}=8 \alpha_{s}^{2} \pi^{4} \quad(p=3) .
$$

Consider the expansion in powers of $q$,

$$
G\left(q, \nu_{i}\right)=\sum_{n} c_{n} q^{n}=1+c_{1} q+c_{2} q^{2}+\ldots
$$

The odd powers of $q$ drop out after integration in $\nu_{i}$. Upon integration in $\tau$, the $n$ power of $q$ produces a pole (for $p=$ 9) or a cut (for other $p$ ) at $\alpha^{\prime} s=2 n$, with $n=2 N$, and $N$ is a positive integer corresponding to the level of the string excitation. This reproduces the spectrum of the free closed superstring, $\alpha^{\prime} M^{2}=4 N$. In conclusion, amplitude $A_{D p}$ has cuts and its imaginary part picks new contributions as $s$ is increased from zero anytime it crosses $\alpha^{\prime} s=$ $4,8,12, \ldots$, etc. This is computed using the formula

$$
\operatorname{Im} \int d x x^{-\alpha} e^{\beta x}=\frac{\pi \beta^{\alpha-1}}{\Gamma(\alpha)} .
$$

This corresponds to having a closed string as intermediate state. The reason of a cut, rather than a pole, is the presence of the Dp-brane. The physical process going on is as follows: The two initial open strings meet into a single open string that propagates on the brane. The end points of this open string then meet and the resulting open string goes into the bulk. Its transverse momentum is absorbed by the brane. It is this intermediate state made of closed string plus brane that produces the cut. By using the optical theorem, we compute the total cross section for producing the closed string going into the bulk:

$$
\sigma(s)=\frac{1}{s} \operatorname{Im} A_{D p} .
$$

The single open string propagating on the brane (just before the closed string is produced) will give rise to poles in the cross section. Such poles have first been observed by [14].

Let us first compute the cross section up to $\alpha^{\prime} s<4$. In this case, we can set $G=1$, since the remaining powers of $q$ give no contribution to the imaginary part. This will give the level $N=0$ contribution, describing the production of (Kaluza-Klein) gravitons. Using the formula (2.8), we obtain

$$
\begin{aligned}
\operatorname{Im} A_{D p} & =\mathcal{N}_{p} K \frac{\pi\left(\frac{\alpha^{\prime} s}{2}\right)^{(7-p) / 2}}{\Gamma\left(\frac{9-p}{2}\right)}\left(\int_{0}^{1} d \nu(2 \sin \pi \nu)^{-\alpha^{\prime} s}\right)^{2} \\
& =\mathcal{N}_{p} K \frac{\pi\left(\frac{\alpha^{\prime} s}{2}\right)^{(7-p) / 2}}{\Gamma\left(\frac{9-p}{2}\right)}\left(\frac{\Gamma\left(\frac{1}{2}-\frac{\alpha^{\prime} s}{2}\right) 2^{-\alpha^{\prime} s}}{\sqrt{\pi} \Gamma\left(1-\frac{\alpha^{\prime} s}{2}\right)}\right)^{2} \\
s & <8 .
\end{aligned}
$$

Using the optical theorem, we have

$$
\sigma_{0}=\mathcal{N}_{p} \frac{1}{s} K \frac{\left(\frac{\alpha^{\prime} s}{2}\right)^{(7-p) / 2}}{\Gamma\left(\frac{9-p}{2}\right)} \frac{2^{-2 \alpha^{\prime} s} \Gamma\left(\frac{1}{2}-\frac{\alpha^{\prime} s}{2}\right)^{2}}{\Gamma\left(1-\frac{\alpha^{\prime} s}{2}\right)^{2}} .
$$

This can be compared with (2.1) when $M=0$. We see that the volume factor $\operatorname{Vol}\left(\Omega_{8-p}\right)$ has emerged automatically, as expected, and also the power of $s^{(7-p) / 2}$ coming from phase space. It agrees with [14] where the amplitude for graviton emission from the brane was computed. The full cross section (2.11) includes - in addition to the graviton-the emission of other Neveu-Schwarz NeveuSchwarz as well as Ramond-Ramond states.

The cross section (2.11) is valid up to $\alpha^{\prime} s=4$. As mentioned before, for $\alpha^{\prime} s>4$ there is a new contribution to the imaginary part due to the opening of a new channel corresponding to $N=1$ level. Including this contribution will give the exact expression for the total cross section up to $\alpha^{\prime} s=8$. For $\alpha^{\prime} s>8$ there will appear a new contribu- 
tion to the imaginary part corresponding to $N=2$ level. The coefficient of the quadratic $q^{2}$ term in $G$ is

$$
\begin{aligned}
c_{2}= & 2\left(\alpha^{\prime} s \cos \left(2 \pi \nu_{12}\right)+1\right)\left(\alpha^{\prime} s \cos \left(2 \pi \nu_{13}\right)+1\right) \\
& +2\left(\alpha^{\prime} s\right)^{2} \sin \left(2 \pi \nu_{12}\right) \sin \left(2 \pi \nu_{13}\right)+2\left(\alpha^{\prime} s\right)^{2}-2 .
\end{aligned}
$$

We will use the basic formula

$$
\begin{aligned}
\int_{0}^{1} d x(\sin (\pi x))^{a}(\cos (\pi x))^{2 b} & =\frac{1}{\pi} B\left(\frac{1}{2}+\frac{a}{2}, \frac{1}{2}+b\right) \\
& =\frac{\Gamma\left(\frac{1}{2}+\frac{a}{2}\right) \Gamma\left(\frac{1}{2}+b\right)}{\pi \Gamma\left(1+\frac{a}{2}+b\right)} .
\end{aligned}
$$

The final result for the cross section is

$$
\sigma=\sigma_{0}(s)+\hat{\theta}\left(\alpha^{\prime} s-4\right) \sigma_{1}(s),
$$

where $\hat{\theta}(x)$ is the step function and

$$
\begin{aligned}
\sigma_{1}(s)= & \mathcal{N}_{p} K \frac{2}{\Gamma\left(\frac{9-p}{2}\right)} \frac{1}{s}\left(\frac{\alpha^{\prime} s}{2}-2\right)^{(7-p) / 2} \\
& \times \frac{2^{-2 \alpha^{\prime} s} \Gamma\left(\frac{1}{2}-\frac{\alpha^{\prime} s}{2}\right)^{2}}{\Gamma\left(1-\frac{\alpha^{\prime} s}{2}\right)^{2}} B, \\
B \equiv & \left(\frac{\left(\alpha^{\prime} s\right)^{2}}{2\left(1-\frac{\alpha^{\prime} s}{2}\right)}+1\right)^{2}+\left(\alpha^{\prime} s\right)^{2}-1 .
\end{aligned}
$$

In conclusion, for the case of a D3-brane, we find the following formula for the total cross section (for an initial state with two gauge vectors):

$$
\begin{gathered}
\sigma_{0}(s)=\pi^{4}\left(\xi_{1}^{2} \xi_{2}^{2}\right) \frac{\alpha_{s}^{2}}{M_{s}^{2}}\left(\frac{s}{M_{s}^{2}}\right)^{3} \frac{2^{-2 \alpha^{\prime} s} \Gamma\left(\frac{1}{2}-\frac{\alpha^{\prime} s}{2}\right)^{2}}{\Gamma\left(1-\frac{\alpha^{\prime} s}{2}\right)^{2}}, \\
\sigma_{1}(s)=8 \pi^{4}\left(\xi_{1}^{2} \xi_{2}^{2}\right) \frac{\alpha_{s}^{2}}{M_{s}^{2}} \frac{s}{M_{s}^{2}}\left(\frac{\alpha^{\prime} s}{2}-2\right)^{2} \\
\times \frac{2^{-2 \alpha^{\prime} s} \Gamma\left(\frac{1}{2}-\frac{\alpha^{\prime} s}{2}\right)^{2}}{\Gamma\left(1-\frac{\alpha^{\prime} s}{2}\right)^{2}} B .
\end{gathered}
$$

\section{LIMITS ON CROSS SECTIONS AND WIDTHS OF OPEN-STRING STATES}

Consider a process $a \rightarrow b$, with a number of resonances in the intermediate channel, with possibly different widths (as this is the typical case in string theory).

In our case, in particular, we have as first open-string resonances: four scalars, one spin 1 and one spin 2 resonances, denoted as $\left|0_{q=1,2,3,4}\right\rangle,\left|n_{1}\right\rangle$ and $\left|n_{2}\right\rangle$, respectively. They occur for $\alpha^{\prime} s=1$ [masses $M_{r}=\left(\alpha^{\prime}\right)^{-1 / 2}$ ] and couple to various helicities (see [7]).

The general cross-section formula for resonances of the same mass is

$$
\sigma=\frac{16}{s} \frac{\pi}{\mathcal{N}} \sum_{j}(2 j+1) \frac{M_{r}^{2} \Gamma_{j a} \Gamma_{j b}}{\left(s-M_{r}^{2}\right)^{2}+\Gamma_{j}^{2} M_{r}^{2}},
$$

where we have indicated as $|a\rangle$ the initial states, as $|b\rangle$ the final ones (in our case, the closed-string states). The sum is over resonances with different spin $j$, whose total decay rate is $\Gamma_{j}\left(\Gamma_{j a, b}\right.$ are partial width). $\mathcal{N}$ is the multiplicity of the initial states, that is the number of possible polarizations times a possible Bose symmetry factor.

This formula can be obtained from unitarity (see the appendix), generalizing a similar formula given in [16].

In general $\Gamma_{j}=\Gamma_{j a}+\Gamma_{j b}+\Gamma_{j, \text { other }}$, so that

$$
\frac{\Gamma_{j, a} \Gamma_{j, b}}{\Gamma_{j}^{2}} \leq \frac{1}{4}
$$

and the bound for $s=M_{r}^{2}$ is

$$
\sigma \leq \frac{4 \pi}{M_{r}^{2} \mathcal{N}} \sum_{j}(2 j+1) .
$$

Considering the various initial states and the related resonances (see below, in the text), we get the bounds: $\left\langle\sigma_{g g}\right\rangle \leq$ $14 \pi / M_{r}^{2}$ for the gluon-gluon initial state and $\left\langle\sigma_{q \bar{q}}\right\rangle \leq$ $18 \pi / M_{r}^{2}$ for the quark-antiquark one.

Using the results of [7], we can actually compute the width of the resonances for $\alpha^{\prime} s=1$.

In the formula (2.17) the width is put to zero. We can compare it for $\alpha^{\prime} s \rightarrow 1$ with (3.1) when we similarly consider $\Gamma_{j} \rightarrow 0$ in the denominator. Putting $\alpha^{\prime} s=1+y$, with $y \rightarrow 0$ we get

$$
\alpha^{\prime} \pi^{4} \alpha_{s}^{2} \frac{1}{\pi} \frac{1}{y^{2}}=\alpha^{\prime} \frac{16 \pi}{\mathcal{N}} \sum_{j}(2 j+1) \alpha^{\prime} \frac{\Gamma_{j a} \Gamma_{j b}}{y^{2}} .
$$

According to [7] the various resonances couple to some specific initial states (we consider QCD particles - gluon $g$ and quark $q$-rather than QED particles as in [7]). Note that the resonances in our case are colorless.

$$
\begin{aligned}
\mathbf{g}_{\mathbf{R}(\mathbf{L})} \mathbf{g}_{\mathbf{R}(\mathbf{L})} \rightarrow\left|\mathbf{0}_{\mathbf{1}}\left(\mathbf{0}_{\mathbf{2}}\right)\right\rangle, & \overline{\mathbf{q}}_{\mathbf{R}(\mathbf{L})} \mathbf{q}_{\mathbf{R}(\mathbf{L})}^{-} \rightarrow\left|\mathbf{0}_{\mathbf{3}},\left(\mathbf{0}_{\mathbf{4}}\right)\right\rangle, \\
\mathbf{g}_{\mathbf{L}} \mathbf{g}_{\mathbf{R}} \rightarrow|\mathbf{2}\rangle, & \overline{\mathbf{q}}_{\mathbf{L}} \mathbf{q}_{\mathbf{R}}^{-} \rightarrow|\mathbf{1}, \mathbf{2}\rangle .
\end{aligned}
$$

Take for instance $g_{R(L)} g_{R(L)}$ : Here $\mathcal{N}_{g g}=1 / 2$ and using $\Gamma_{01}\left(g_{L(R)} g_{L(R)}\right)$ from [7] we find

$$
\sqrt{\alpha^{\prime}} \Gamma_{01 b}=\sqrt{\alpha^{\prime}} \Gamma_{02 b}=\frac{\pi^{2}}{8} \alpha_{s}
$$

Similarly we get

$$
\begin{gathered}
\sqrt{\alpha^{\prime}} \Gamma_{03 b}=\sqrt{\alpha^{\prime}} \Gamma_{04 b}=\frac{\pi^{2}}{8} \alpha_{s}, \quad \sqrt{\alpha^{\prime}} \Gamma_{1 b}=\frac{\pi^{2}}{12} \alpha_{s}, \\
\sqrt{\alpha^{\prime}} \Gamma_{2 b}=\frac{\pi^{2}}{4} \alpha_{s} .
\end{gathered}
$$

We now have to estimate the total widths, by summing over all possible decay channels. In principle we should 
then include also weakly and QED interacting particles, but we neglect this contribution since the relevant coupling constant is 1 order of magnitude less than $\alpha_{s}$. In this way we find:

$$
\begin{gathered}
\Gamma_{01}=\Gamma_{02}=\left(2+\frac{\pi^{2}}{8}\right) \alpha_{s} M_{s}, \\
\Gamma_{03}=\Gamma_{04}=\left(9+\frac{\pi^{2}}{8}\right) \alpha_{s} M_{s}, \\
\Gamma_{1}=\left(\frac{9}{2}+\frac{\pi^{2}}{12}\right) \alpha_{s} M_{s}, \quad \Gamma_{2}=\left(\frac{13}{10}+\frac{\pi^{2}}{4}\right) \alpha_{s} M_{s}, \\
M_{s}=\frac{1}{\sqrt{\alpha^{\prime}}} .
\end{gathered}
$$

The cross sections. - In principle we should also know the widths of the higher open-string resonances $\left(\alpha^{\prime} s=\right.$ $3,5,7)$. As an estimate we simply use the same width for all of them, obtained by averaging over the various widths for $\alpha^{\prime} s=1$, for the initial states $|g g\rangle$ or $|q \bar{q}\rangle$.

Numerically we take $\left(\alpha_{s}=0.1\right)$

$$
\begin{aligned}
\Gamma_{g g} & =\frac{\left(\Gamma_{01}+\Gamma_{02}+\Gamma_{2}\right)}{3}=\frac{1}{3}\left(\frac{53}{10}+\frac{\pi^{2}}{2}\right) \frac{\alpha_{s}}{\sqrt{\alpha^{\prime}}}=0.34 M_{s}, \\
\Gamma_{q \bar{q}} & =\frac{\left(\Gamma_{03}+\Gamma_{04}+\Gamma_{1}+\Gamma_{2}\right)}{4}=\frac{1}{4}\left(\frac{119}{5}+\frac{7 \pi^{2}}{12}\right) \frac{\alpha_{s}}{\sqrt{\alpha^{\prime}}} \\
& =0.74 M_{s} .
\end{aligned}
$$

These are the values we have used in plotting the cross sections with formula (2.14) with the substitution (5.1). Note that $\Gamma_{q \bar{q}}$ is larger than $\Gamma_{g g}$ due to the sum over generations and flavors.

A further comment: the bound (3.3) is independent of the normalization (2.6) and also our results will be less sensitive to the normalization than one could have expected. In fact, a change in the normalization of the lefthand side of (3.4) will affect the determination of $\Gamma_{j b}$ [in the numerator of (3.1)], and in turn the total width $\Gamma_{j}$ (in the denominator [of (3.1)]). Thus there will be some compensation, and even more after integrating the cross section over an interval of the total energy.

\section{CROSS SECTION FOR MISSING-ENERGY PROCESSES IN COLLIDERS}

In [4], the cross section for the process of Kaluza-Klein graviton $G$ emission in electron-positron annihilation, $e^{+} e^{-} \rightarrow \gamma G$, was determined. This process gives a missing-energy signature, which has been used by LEP2 experiments [17] to put constraints on the size of extra dimensions. This process was further studied in [5], in particular, in [6,7], where the string-theory corrections were computed.

In general, a process gluon + gluon (or fermion + antifermion) $\rightarrow$ gluon + missing transverse momentum can have many contributions of different origin. In string theory, in addition to the graviton considered in [6,7], there are also states from the Neveu-Schwarz Neveu-Schwarz and Ramond-Ramond sectors which contribute to the cross section. In particular, there are massless NS-NS and R-R states which in four dimensions are scalar or vector particles. They are expected to get a mass because a potential is generated for the scalars and the U(1) gauge symmetry is also broken by mechanisms which are strongly model dependent, involving fluxes, compactification scales and details of supersymmetry breaking. Their expected masses are also model dependent. Here we treat them as massless, which is justified if their masses are much smaller than the string scale.

Here we will compute the cross section which will include, in addition to the process of Kaluza-Klein graviton emission studied in earlier works, emission of R-R states and emission of closed-string states of the first excited. The contribution from the first excited closed-string states appears for $\alpha^{\prime} s>4$.

Thus the total cross section obtained here represents the full cross section for missing-energy processes that takes into account all possible string state production up to $s<$ $8 M_{s}^{2}$. These results can be used to put new constraints on the string scale.

We consider the amplitude

$$
A_{C}=A(a+b \rightarrow C+c)
$$

where $a, b, g$ are gluons or quarks and $C$ is a (missing) closed-string state. The closed-string state $C$ has momentum $p_{\hat{\mu}}=\left(E_{C}, \vec{p}_{C}, \Delta_{i}\right), \hat{\mu}=0, \ldots, 9, i=4, \ldots, 9$, with $\Delta_{i}$ being the components transverse to the brane. Its mass in ten dimensions is $M_{C}^{2}=E_{C}^{2}-\vec{p}_{C}^{2}-\Delta^{2}$. This corresponds to a four-dimensional mass equal to $M_{4}^{2} \equiv$ $E_{C}^{2}-\vec{p}_{C}^{2}=M_{C}^{2}+\Delta^{2}$. Since the six extra dimensions are compactified on a 6-torus, $\Delta_{i}$ are quantized in units of inverse radius of the toroidal directions. When the extra dimensions are very large compared to the $\mathrm{TeV}$ scale we can consider $\Delta_{i}$ as a continuous variable and for simplicity we make this assumption here. We have also considered the case of two large and four small extra dimensions, and obtained very similar results, reported in Sec. V.

For center of mass energies $\sqrt{s}<2 M_{s}$, the particle $C$ can only be a massless closed-string state. The cross section picks an important extra contribution when $\sqrt{s}>2 M_{s}$ coming from all bosonic closed-string states of the $N=1$ level, with mass $M=2 M_{s}$. The final cross section will be a function of $s, M_{C}$, the missing energy $E_{C}$ and of the scattering angle $\theta$. It is important to note that in ordinary four-dimensional processes, the four-point amplitudes depend on two kinematical variables, e.g. $s$ and $t$, or $s$ and $\theta$. 
The reason of the dependence on $s, E_{C}$ and $\theta$ in the present case is due to the momentum nonconservation in the directions transverse to the brane.

We will consider as initial states in the collision: (i) two gluons, (ii) quark and antiquark, (iii) quark and gluon (an initial state of two quarks cannot produce a colorless closed-string state). In cases (i),(ii) the final particle $c$ is a gluon, in the case (iii) it is a quark.

We begin by illustrating the general computation taking the case (i).

We consider the CM frame, with momenta and polarization tensors as follows:

$$
\begin{array}{r}
p_{\mu}^{a}=\left(p_{a}, \vec{p}_{a}\right), \quad p_{\mu}^{b}=\left(p_{a},-\vec{p}_{a}\right), \\
p_{\mu}^{a} p^{a \mu}=p_{\mu}^{b} p^{b \mu}=0, \\
p_{\mu}^{c}=\left(p_{c}, \vec{p}_{c}\right), \quad P_{\hat{\mu}}^{C}=\left(E_{C},-\vec{p}_{c}, \Delta_{i}\right), \\
p_{\mu}^{c} p^{c \mu}=0, \quad E_{C}^{2}-\vec{p}_{c}^{2}-\Delta^{2}=M_{C}^{2}, \\
E_{C}+p_{c}=2 p_{a}, \\
\vec{\zeta}^{i} \cdot \vec{p}^{i}=0, \quad \zeta_{0}^{i}=0, \quad i=a, b, c .
\end{array}
$$

We consider the case in which the gluon $c$ is soft, i.e. it carries an energy much less than the string scale. More precisely, we assume $p_{c} \ll 1 / \alpha^{\prime} p_{a}$. In the CM frame, $s=$ $4 p_{a}^{2}$. Since the string-theory corrections are significant for $s>O\left(M_{s}\right)$ and $M_{s}>O(1 \mathrm{TeV})$, the gluon $c$ could even carry energies as large as, say, $0.1 \mathrm{TeV}$, and the present approximation will miss only corrections of few percent. Given the bremsstrahlung character of the emission process, the approximation of a soft emitted gluon will be accurate for most missing-energy events that will appear in a collider (this is confirmed by the cross section obtained below, which decreases like $1 / p_{c}$ ).

We will call, then, $V_{C}$ the vertex (to be used in Feynman graphs) for producing the state $C$ from two (real or virtual) gluons $i$ and $j$ :

$$
V_{C}=\zeta_{\mu}^{i} \zeta_{\nu}^{j} T^{\mu \nu A} \epsilon_{A}
$$

where $\zeta^{i, j}$ are the gluon polarization and $\epsilon$ is the polarization of the closed-string state equipped with whichever indices $A$ are necessary.

Note that

$$
\begin{aligned}
\sum_{\epsilon} \int\left|V_{C}\right|^{2} \frac{d \Omega_{5} \Delta^{4}}{2(2 \pi)^{5}} & =2 \operatorname{Im}\left[A_{\mathrm{D} 3}\right] \\
& =2 \hat{s}\left(\sigma_{0}(\hat{s})+\theta(\hat{s}-4) \sigma_{1}(\hat{s})\right) .
\end{aligned}
$$

Here $A_{\mathrm{D} 3}$ is Eq. (2.2) for the case $p=3, \vec{\Delta}$ is the closed state K-K momentum transverse to the brane and $\hat{s}=$ $M_{C}^{2}+\Delta^{2}=s-2 p_{c} \sqrt{s}$ is the square mass including the $\mathrm{K}-\mathrm{K}$ momentum.
The cross section for the process $a+b \rightarrow C+c$ will be

$$
\begin{aligned}
d \sigma= & \frac{1}{2 s} \int\left|A_{C}\right|^{2} \frac{d^{6} \Delta d^{3} p_{C}}{2(2 \pi)^{9} E_{C}} \frac{d^{3} p_{c}}{2(2 \pi)^{3} p_{c}}(2 \pi)^{3} \delta^{3}\left(\vec{p}_{C}+\vec{p}_{c}\right) \\
& \times(2 \pi) \delta\left(2 p_{a}-p_{c}-E_{C}\right) \\
= & \frac{1}{2 s} F \frac{d^{3} p_{c}}{2(2 \pi)^{3} p_{c}}
\end{aligned}
$$

where

$$
\begin{aligned}
F & =\int\left|A_{C}\right|^{2} \frac{d^{6} \Delta}{2(2 \pi)^{6} E_{C}}(2 \pi) \delta\left(2 p_{a}-p_{c}-E_{C}\right) \\
& =\int\left|A_{C}\right|^{2} \frac{d \Omega_{5} \Delta^{4}}{2(2 \pi)^{5}} .
\end{aligned}
$$

By dimensional reasons $[F]=p^{-2}$ and we look for the leading terms, that is, the ones proportional to $p_{c}^{-2}$ since $p_{c}$ is the smallest energy scale. These terms can only come from the (modulus square of the sum of the) Feynman graphs having a pole in the Mandelstam variables $t=$ $-2 p_{a} \cdot p_{c}, u=-2 p_{b} \cdot p_{c}$, due to an intermediate virtual gluon. Therefore we compute $A_{C}$ by considering only those two graphs (see Fig. 1, I and II).

The resulting cross section will be of the form

$$
d \sigma=\frac{1}{s} f d \Omega_{2} \frac{d p_{c}}{p_{c}},
$$

where $f=f\left(s, \hat{s}, \alpha^{\prime}\right)$ is dimensionless. Notice the factor $d p_{c} / p_{c}$ characteristic of a bremsstrahlung process, in this case the bremsstrahlung of the gluon $c$ which is the soft particle in our process.

Take for instance the $t$-channel graph (see Fig. 1, I)

$$
V_{a \rightarrow c+e} \cdot \text { Propagator }_{e} \cdot V_{C}
$$

where Propagator ${ }_{e} \sim 1 / p_{e}^{2}$ and $V_{C}$ is the vertex for $b+$ $e \rightarrow C$ defined in (4.6).

The polarization of $e$ will be computed in terms of the polarizations and the momenta of $a$ and $c$ by the YM vertex $V(\mathrm{YM})_{a \rightarrow c+e}$. One can check that the resulting polarization satisfies $\zeta_{e} \cdot p_{e}=0$.
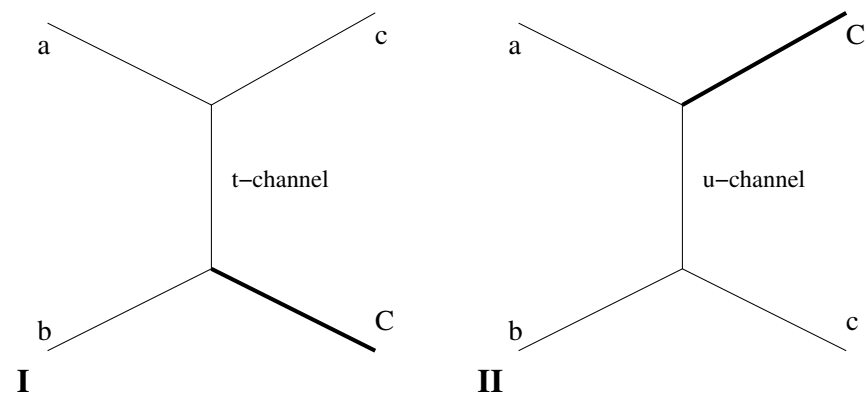

FIG. 1. $t$ and $u$ channels for the process: $a+b \rightarrow c+C$ (missing, closed-string state). 
We get:

$$
A_{C}=-\frac{\zeta_{b \mu} \zeta_{e \nu} T^{\mu \nu A} \epsilon_{A}}{2 p_{a} p_{c}} .
$$

Note that $\left|p_{e}^{2}\right|=\left|\left(p_{a}-p_{c}\right)^{2}\right|=2 p_{a} p_{c} \ll 1 / \alpha^{\prime}$ and therefore, since the scale is set here by $1 / \alpha^{\prime}$, we can take $V_{C}$ to be the vertex to be computed in string theory for massless $b$ and $e$. Thus Eq. (4.7) holds.

By inserting $\left|A_{C}\right|^{2}$ in (4.9) we get

$$
F=W \cdot 2 \operatorname{Im}\left[A_{\mathrm{D} 3}\right]
$$

where $W$ depends on $p_{c}$ and $\theta$ and will be explicitly computed below for the various cases (i)-(iii).

Therefore

$$
d \sigma_{C}=\frac{\hat{s}}{s}\left(\sigma_{0}+\hat{\theta}(\hat{s}-4) \sigma_{1}\right) W \frac{d^{3} p_{c}}{2(2 \pi)^{3} p_{c}} .
$$

In our approximation $\alpha^{\prime}(s-\hat{s}) \ll 1$ and thus we can take $\sigma_{0,1}$ to depend on $s$. Therefore the only dependence on $p_{c}, \theta$ is inside $W$.

(i) Gluon-gluon initial state. - We recall the kinematical factor $K(2.4)$

$$
K=\left(\alpha^{\prime} s\right)^{2}\left(\zeta^{1} \cdot \zeta^{4}\right)\left(\zeta^{2} \cdot \zeta^{3}\right)
$$

for the amplitude $A_{\mathrm{D} 3}=g+g \rightarrow$ closed string $\rightarrow g+g$ (forward scattering corresponds to $1 \rightarrow 4,2 \rightarrow 3$ ).

We use $\zeta^{a}=\zeta_{\mu s}^{a}$ to indicate the particle $a$ with color $s$, etc. For the $(t, u)$ channel we get

$$
\begin{aligned}
\zeta_{e \rho}^{e}= & \zeta_{\mu s}^{a, b} \zeta_{\nu r}^{c} V(\mathrm{YM})_{s r e}^{\nu \mu \rho} \\
= & g_{\mathrm{YM}} f_{s r e}\left(\zeta_{s}^{a, b} \cdot \zeta_{r}^{c}\left(p_{a, b}+p_{c}\right)_{\rho}-2 p_{c} \cdot \zeta_{s}^{a, b} \zeta_{\rho r}^{c}\right. \\
& \left.-2 p_{a, b} \cdot \zeta_{r}^{c} \zeta_{\rho s}^{a, b}\right) .
\end{aligned}
$$

We keep only the last term of this expression since the others give subleading contributions (see the contractions in the following formulas). Thus we define:

$$
\begin{gathered}
\zeta_{\mu e}^{t, u}=-g_{\mathrm{YM}}\left(2 p_{a, b} \cdot \zeta_{r}^{c}\right) \hat{\zeta}_{\mu e}^{t, u} \quad\left(\zeta_{\mu s}^{a, b} f_{s r e} \equiv \hat{\zeta}_{\mu e}^{t, u}\right), \\
t, u=p_{(t, u)}^{2}, \quad p_{(t, u) \mu}=p_{(a, b) \mu}-p_{c \mu} .
\end{gathered}
$$

Note that in the vertex producing the closed-string state, the color indices of the two gluons are contracted together, since the closed state is colorless (technically this is due to the Chan-Paton rules [7]). We have (since $p_{b} \cdot \zeta^{c}=-p_{a}$. $\left.\zeta^{c}\right)$

$$
\begin{aligned}
\sum_{\epsilon} \int & \frac{d \Omega_{5} \Delta^{4}}{2(2 \pi)^{5}} g_{\mathrm{YM}}^{2} 4\left(p_{a} \cdot \zeta^{c}\right)^{2} \\
& \times\left|\left(\frac{\hat{\zeta}_{\mu e}^{t} \zeta_{\nu s}^{b} \delta_{e s}}{t}-\frac{\hat{\zeta}_{\mu e}^{u} \zeta_{\nu s}^{a} \delta_{e s}}{u}\right) T^{\mu \nu A} \epsilon_{A}\right|^{2} \\
= & 2 \operatorname{Im}\left[A_{\mathrm{D} 3}\right]
\end{aligned}
$$

where in $\operatorname{Im}\left[A_{\mathrm{D} 3}\right]$ we have to replace $\left(\zeta_{1} \cdot \zeta_{4}\right)\left(\zeta_{2} \cdot \zeta_{3}\right)$ with the term $W$

$$
\begin{aligned}
W= & g_{\mathrm{YM}}^{2} 4\left(p_{a} \cdot \zeta_{r}^{c}\right)^{2}\left(\frac{\left(\zeta_{s}^{a} \cdot \zeta_{s^{\prime}}^{a}\right)\left(\zeta_{h}^{b} \cdot \zeta_{h^{\prime}}^{b}\right)}{t^{2}}\right. \\
& \left.+\frac{\left(\zeta_{s}^{b} \cdot \zeta_{s^{\prime}}^{b}\right)\left(\zeta_{h}^{a} \cdot \zeta_{h^{\prime}}^{a}\right)}{u^{2}}-2 \frac{\left(\zeta_{s}^{a} \cdot \zeta_{h^{\prime}}^{a}\right)\left(\zeta_{h}^{b} \cdot \zeta_{s^{\prime}}^{b}\right)}{t u}\right) f_{s r h} f_{s^{\prime} r h^{\prime}}
\end{aligned}
$$

here $\zeta_{s}^{b} \cdot \zeta_{s^{\prime}}^{b}=\zeta_{\mu s}^{b} \zeta_{\mu s^{\prime}}^{b}$ and the sum is over colors $s, s^{\prime}, h, h^{\prime}$ at fixed $c$.

The last interference term corresponds to the amplitude (2.2) with $\zeta^{t}+\zeta^{b} \rightarrow \zeta^{a}+\zeta^{u}$ which is forward in the sense that $\left(p^{t}-p^{a}\right)^{2}=0,\left(p^{b}-p^{u}\right)^{2}=0$.

We average over $\zeta^{a}, \zeta^{b}$ and sum over $\zeta^{c}$ getting for $\mathrm{SU}(N)$

$$
W=\frac{g_{\mathrm{YM}}^{2} N}{\left(N^{2}-1\right)} \frac{1}{p_{c}^{2}} \frac{4}{\sin ^{2}(\theta)} .
$$

In conclusion we get $^{2}$ for SU(3), after integrating over $\phi$,

$$
d \sigma=\left(\sigma_{0}+\hat{\theta}\left(\alpha^{\prime} s-4\right) \sigma_{1}\right) \alpha_{s} \frac{2}{\pi} \frac{3}{8} \frac{d p_{c}}{p_{c}} \frac{d \theta}{\sin (\theta)} .
$$

(ii) Quark-antiquark initial state. - We recall the kinematical factor $K(2.5)$

$$
K=\alpha^{\prime} s 2 \alpha^{\prime}\left(\bar{v}_{2 s} \gamma^{\mu} v_{3 r}\right)\left(\bar{u}_{4 r} \gamma_{\mu} u_{1 s}\right),
$$

for the amplitude $A_{\mathrm{D} 3}=q+\bar{q} \rightarrow$ closed string $\rightarrow q+\bar{q}$, that is $u_{1}\left(k_{1}\right)+\bar{v}_{2}\left(k_{2}\right) \rightarrow u_{4}\left(k_{4}\right)+\bar{v}_{3}\left(k_{3}\right)$ (forward is $1 \rightarrow$ 4 and $2 \rightarrow 3$ ). We have indicated the color indices $s, r$. We normalize the spinors by requiring that the current is the same as in the bosonic case: $\bar{u} \gamma^{\mu} u=p^{\mu}$. With this normalization the Dirac average is $\left\langle u_{s} \bar{u}_{r}\right\rangle=\left(p \cdot \gamma \delta_{s r}\right) / 4$.

Our initial state is $u^{a}, \bar{v}^{b}$ and we call $u^{t}\left(\bar{v}^{u}\right)$ the intermediate quark (antiquark). Let us call $\bar{v} V^{A} u \epsilon_{A}$ the vertex for producing the closed state from $q+\bar{q}$. The leading amplitude for $F$ comes from the two graphs with a pole in the $t$ and $u$ channels

$$
A_{t}=\bar{v}^{b} V^{A} u^{t} \epsilon_{A}, \quad A_{u}=\bar{v}^{u} V^{A} u^{a} \epsilon_{A} .
$$

Here

$u^{t}=g_{\mathrm{YM}} \frac{\left(p_{t} \cdot \gamma\right)\left(\zeta_{c} \cdot \gamma\right) t_{c} u^{a}}{t} \quad$ with $p_{t}=p_{a}-p_{c}$,

$\overline{\boldsymbol{v}}^{u}=-g_{\mathrm{YM}} \frac{\overline{\boldsymbol{v}}^{b} t_{c} \zeta_{c} \cdot \gamma p_{u} \cdot \gamma}{u} \quad$ with $p_{u}=p_{b}-p_{c}$.

$t_{c}$ is a $\mathrm{SU}(N)$ matrix in the fundamental representation $\operatorname{Tr}\left(t_{c}^{2}\right)=1 / 2$. Now we have

$$
\sum_{\epsilon} \int \frac{d \Omega_{5} \Delta^{4}}{2(2 \pi)^{5}}\left|A_{t}+A_{u}\right|^{2}=2 \operatorname{Im}\left[A_{\mathrm{D} 3}\right],
$$

\footnotetext{
${ }^{2}$ We have checked that our result coincides with the expression obtained using the amplitude in Eq. (4.14) of [6], in the particular case considered there, by taking the relevant limit and normalization.
} 
where in $\operatorname{Im}\left[A_{\mathrm{D} 3}\right]$ we have to replace $\left(\bar{u}_{2 s} \gamma^{\mu} u_{3 r}\right)\left(\bar{u}_{4 r} \gamma_{\mu} u_{1 s}\right)$ with the term

$$
\begin{aligned}
Q= & \left(\overline{\boldsymbol{v}}_{s}^{b} \gamma^{\mu} \boldsymbol{v}_{r}^{b}\right)\left(\bar{u}_{r}^{t} \gamma_{\mu} u_{s}^{t}\right)+\left(\bar{u}_{s}^{a} \gamma^{\mu} u_{r}^{a}\right)\left(\overline{\boldsymbol{v}}_{r}^{u} \gamma_{\mu} \boldsymbol{v}_{s}^{u}\right) \\
& +2\left(\overline{\boldsymbol{v}}_{s}^{b} \gamma^{\mu} \boldsymbol{v}_{r}^{u}\right)\left(\bar{u}_{r}^{a} \gamma_{\mu} u_{s}^{t}\right) .
\end{aligned}
$$

The last interference term corresponds to the amplitude (2.2) with $u^{t}\left(p_{t}\right) \rightarrow u^{a}\left(p_{a}\right) v^{b}\left(p_{b}\right) \rightarrow v^{u}\left(p_{u}\right)$, which is forward: $\hat{t}=\left(p_{t}-p_{a}\right)^{2}=\left(p_{b}-p_{u}\right)^{2}=p_{c}^{2}=0$.

Now we substitute the expressions for $u_{t}, v_{u}$ and we make the Dirac and color average over our initial states. We find:

$$
\begin{aligned}
\left\langle\left(\bar{v}_{s}^{b} \gamma^{\mu} v_{r}^{b}\right)\left(\bar{u}_{r}^{t} \gamma_{\mu} u_{s}^{t}\right)\right\rangle= & \frac{g^{2}}{t^{2}} \frac{\operatorname{Tr}\left(t_{c}^{2}\right)}{N^{2}} p_{2}^{\mu}\left\langle\bar{u}^{a} \zeta_{c} \cdot \gamma p_{t} \cdot \gamma \gamma_{\mu} p_{t}\right. \\
& \left.\cdot \gamma \zeta_{c} \cdot \gamma u^{a}\right\rangle \\
& \rightarrow \frac{g^{2}}{N^{2} 8 t^{2}} \frac{s}{2} 16\left(p_{1} \cdot \zeta_{c}\right)^{2} .
\end{aligned}
$$

We have kept only the leading terms (those proportional to four powers of $p_{a}=p_{b}$ ) noting that $p_{t} \cdot \zeta_{c} \sim p_{a} \cdot \zeta_{c}, p_{b}$. $p_{t} \sim p_{a} \cdot p_{b}$. Similarly

$$
\left\langle\left(\bar{u}_{s}^{a} \gamma^{\mu} u_{r}^{a}\right)\left(\bar{v}_{r}^{u} \gamma_{\mu} v_{s}^{u}\right)\right\rangle \rightarrow \frac{g^{2}}{N^{2} 8 u^{2}} \frac{s}{2} 16\left(p_{1} \cdot \zeta_{c}\right)^{2} .
$$

For the last term we get

$$
\left\langle\overline{\boldsymbol{v}}_{s}^{a} \gamma^{\mu} \boldsymbol{v}_{r}^{u}\right\rangle\left\langle\bar{u}_{r}^{a} \gamma_{\mu} u_{s}^{t}\right\rangle \rightarrow \frac{g^{2}}{N^{2} 32 t u} \frac{s}{2} 64\left(p_{1} \cdot \zeta_{c}\right)^{2} .
$$

The final result is

$$
\begin{aligned}
\alpha^{\prime} s 2 \alpha^{\prime} Q & =\left(\alpha^{\prime} s\right)^{2} 2 \frac{g^{2}}{N^{2}}\left(p_{1} \cdot \zeta_{c}\right)^{2}\left(\frac{1}{t^{2}}+\frac{1}{u^{2}}+\frac{2}{t u}\right) \\
& =\left(\alpha^{\prime} s\right)^{2} 2 \frac{g^{2}}{N^{2}} \frac{\left(p_{1} \cdot \zeta_{c}\right)^{2}}{4 p_{1}^{2} p_{c}^{2}} \frac{4}{\left(1-\cos ^{2}(\theta)\right)^{2}} .
\end{aligned}
$$

We can express the result of the quark-antiquark case by saying that: in $\operatorname{Im}\left[A_{\mathrm{D} 3}\right]$ we have to replace $\left(\zeta_{1} \cdot \zeta_{4}\right)\left(\zeta_{2} \cdot \zeta_{3}\right)$ (case of the gluons) with the term $W$, which after summing over the polarizations and the colors of the emitted gluon is

$$
W=\frac{g^{2}\left(N^{2}-1\right)}{N^{2}} \frac{2}{p_{c}^{2} \sin ^{2} \theta} .
$$

In conclusion, after integrating over $\phi$, in the case of SU(3) we get

$$
d \sigma=\left(\sigma_{0}+\hat{\theta}\left(\alpha^{\prime} s-4\right) \sigma_{1}\right) \alpha_{s} \frac{8}{9 \pi} \frac{d p_{c}}{p_{c}} \frac{d \theta}{\sin \theta} .
$$

(iii) Quark-gluon initial state. - We find that in this case $d \sigma$ is subleading with respect to (i) and (ii) by a factor $p_{c} / p_{a}$. This is due to the fact that in the $t$ channel we get a gluon $\zeta_{\mu}^{t} \sim \bar{u}\left(p_{c}\right) \gamma_{\mu} u\left(p_{b}\right)$ and in the $u$ channel we get a fermion $u^{u} \sim \gamma \cdot p_{u} \gamma \cdot \zeta^{a} u\left(p_{c}\right)$. Thus in the average over $u\left(p_{c}\right)$ there is an extra power of $p_{c}$ in the numerator.
Integrated cross section. - The results for $d \sigma$ are the same, up to a constant which is also numerically rather similar, for the cases (i) and (ii), see Eqs. (4.21) and (4.32). In our approximation $\sigma_{0,1}$ are independent of $p_{c}$ and $\theta$. Therefore we can easily perform the integration on those variables, in particular, for a range $\Delta \theta$ around $\pi / 2$ such that $\sin (\theta) \sim 1$. We get

$$
\sigma=\mathcal{A} \frac{\alpha_{s}}{\pi}\left(\sigma_{0}+\hat{\theta}\left(\alpha^{\prime} s-4\right) \sigma_{1}\right) \log \frac{p_{c}^{\max }}{p_{c}^{\min }} \Delta \theta,
$$

where $\mathcal{A}$ is $6 / 8$ for case (i) and $8 / 9$ for case (ii).

\section{ANALYSIS OF THE PARTON CROSS SECTIONS}

In the previous section we have evaluated the cross section for the case in which the final state contains, besides the "missing" closed string, a gluon with transverse momentum (which is small as compared to $M_{s}$ ), as a function of the center of mass energy squared up to $s=$ $8 M_{s}^{2}$. The cross section is proportional to (2.14), (2.17), and (2.18) and has poles coming from the Gamma functions at $\alpha^{\prime} s=1,3,5,7$, representing open-string resonances. The poles have been regularized by including finite widths of the open-string resonances [of order $\Gamma=O\left(\alpha_{s} M_{s}\right)$ ] according to the following recipe. We rewrite the factor $\Gamma\left(\frac{1}{2}-\frac{\alpha^{\prime} s}{2}\right)$ in (2.17) and (2.18), which contains the poles, as:

$$
\begin{aligned}
\left|\Gamma\left(\frac{1}{2}-\frac{\alpha^{\prime} s}{2}\right)\right|^{2} & =\frac{\pi^{2}}{\Gamma\left(\frac{1}{2}+\frac{\alpha^{\prime} s}{2}\right)^{2}} \frac{1}{\cos ^{2}\left(\pi \frac{\alpha^{\prime} s}{2}\right)} \\
& \rightarrow \frac{\pi^{2}}{\Gamma\left(\frac{1}{2}+\frac{\alpha^{\prime} s}{2}\right)^{2}} \frac{c(\Gamma)}{\left|\cos \left(\frac{\pi}{2}\left(\alpha^{\prime} s+i \alpha^{\prime} \sqrt{s} \Gamma\right)\right)\right|^{2}},
\end{aligned}
$$

with $c(\Gamma)=\left(4 / \alpha^{\prime} \Gamma^{2} \pi^{2}\right) \sinh ^{2}\left(\frac{\pi}{2} \sqrt{\alpha^{\prime}} \Gamma\right)$ in order to match the left-hand side of (3.4) when $y^{2} \rightarrow y^{2}+\alpha^{\prime} \Gamma^{2}$ for $y \rightarrow 0$.

The widths of open-string resonances $\alpha^{\prime} s=2 n+1$, with $n \geq 1$, are not known. We have taken in (5.1) the numerical value of $\Gamma$ at $n=0$ estimated in Sec. III, considering for all the different resonances the same averaged value (either $\Gamma_{g g}$ or $\Gamma_{q \bar{q}}$ ) depending on the initial state, consisting of two gluons $(\mathrm{gg})$ or fermion and antifermion $(q \bar{q})$. One expects more massive resonances to have larger widths since at higher level there is more phase space and more decay channels available. This fact is qualitatively taken into account by our prescription (5.1), which near a pole $\alpha^{\prime} s=2 n+1$ reads

$$
\left|\Gamma\left(\frac{1}{2}-\frac{\alpha^{\prime} s}{2}\right)\right|^{2} \rightarrow \frac{4}{n ! \alpha^{\prime} \Gamma^{2}}\left(\frac{\sinh \left(\frac{\pi}{2} \sqrt{\alpha^{\prime}} \Gamma\right)}{\sinh \left(\frac{\pi}{2} \sqrt{2 n+1} \sqrt{\alpha^{\prime}} \Gamma\right)}\right)^{2}
$$

so that the width of the resonance effectively increases as $n$ is increased.

We have seen that the differential cross sections for gluon-gluon Eq. (4.21) and quark-antiquark Eq. (4.32) 
are proportional to $\sigma=\sigma_{0}+\hat{\theta}\left(\alpha^{\prime} s-4\right) \sigma_{1}$, the difference being the numerical factor in front and, more importantly, the widths. In Fig. 2 we show $p_{c} \sin \theta \times d \sigma / d p_{c} d \theta$ for both cases, in units of $\alpha^{\prime}$, as a function of $\alpha^{\prime} s$ ( $s$ being the square of the CM energy). Note that, in the range of validity of our approximations, this is independent of $p_{c}$ and $\theta$. We take $\alpha_{s}=0.1$.

As explained in the previous section, the case gluon-quark $\rightarrow$ closed-string(missing) + quark is subleading and quark-quark $\rightarrow$ closed-string(missing) + any is forbidden by color symmetry.

In Fig. 3 we show the gluon-gluon cross section in the case when two of the extra dimensions are large and the remaining four dimensions are small with radius $R=\sqrt{\alpha^{\prime}}$. The relevant cross-section formula is obtained by replacing, in the phase space factor in (2.1), the integrals over momenta in the small compactified dimensions by the sums over the Kaluza-Klein modes. This in turns amounts to simply substitute in formula (4.33)

$$
\left(s-M^{2}\right)^{2} \hat{\theta}\left(s-M^{2}\right) \rightarrow \frac{2}{\pi^{2} R^{4}} \sum_{n_{1}, n_{2}, n_{3}, n_{4}} \hat{\theta}\left(s-\sum_{j=1}^{4} \frac{n_{j}^{2}}{R^{2}}-M^{2}\right),
$$

which produces a number of discontinuities in the cross section.

In order to make predictions for the measurable $p p$ cross section which is relevant for LHC, the parton cross sections given here have to be convoluted with the parton distribution functions. In addition, one would need to study the dependence on physical observables for jets. The main effect of the parton distribution functions will be to smooth out the various structures of the parton cross sections, like peaks and discontinuities. It would be interesting to investigate whether the possible effects of the string resonances could be recognized from the data. This requires a separate analysis by collider phenomenology experts.

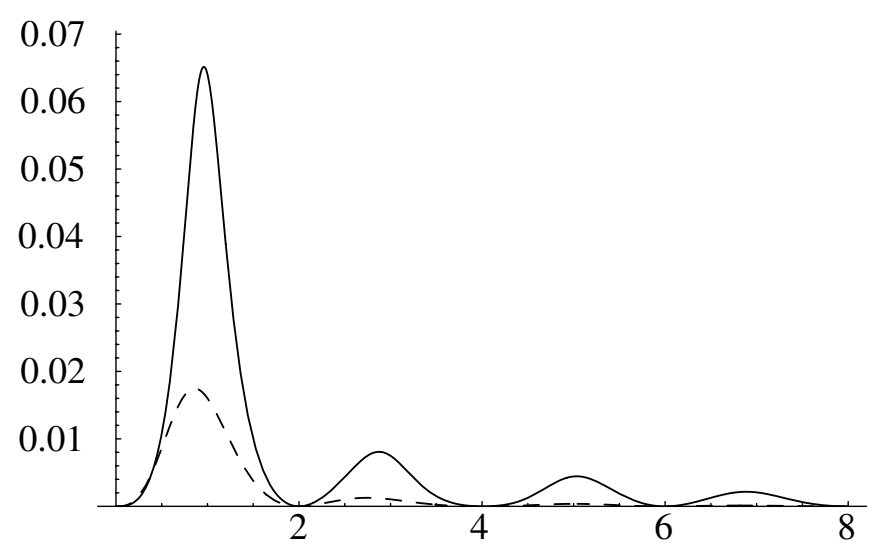

FIG. 2. Process gluon + gluon $\rightarrow$ closed-string $($ missing $)+$ gluon (continuous line) and quark + antiquark $\rightarrow$ closed-string(missing) + gluon (dashed line), cross section in units of $\alpha^{\prime}$, as a function of $\alpha^{\prime} s$.

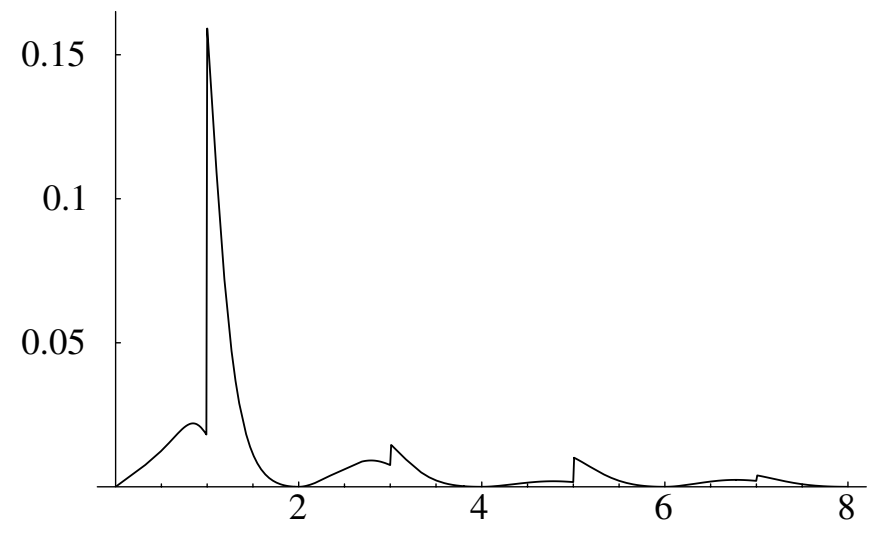

FIG. 3. Process gluon + gluon $\rightarrow$ closed-string(missing $)+$ gluon in the case of two large and four small (with radii $R=$ $\sqrt{\alpha^{\prime}}$ ) extra dimensions.

\section{CONCLUDING REMARKS}

Missing-energy processes provide an efficient way to look for large extra dimensions. In brane world string models, there are many possible processes leading to missing energy, coming from the large number of string excitations. Here we have described a simple method to incorporate in a closed form all string-theory contributions to processes of missing energy by expressing the cross section of the process $g g \rightarrow g+$ missing (or $q \bar{q} \rightarrow g+$ missing) in terms of the imaginary part of a nonplanar amplitude. We have also made an estimate of the decay widths.

The cross section at the peaks is very sensitive to the decay widths. In realistic models the widths could be smaller than those presented here, because after supersymmetry breaking some of the $N=0$ states become massive and the phase space for decaying into them is reduced. As a result, the size of the cross section could be bigger.

Another interesting feature of the total cross section is its smooth behavior after going through the threshold for production of closed-string states of level number $N=1$. We have checked that for $\alpha^{\prime} s>4$ the dominant contribution comes from the $N=1$ states, while the contribution of the $N=0$ states is significantly smaller. In general, the string-theory cross section is of the form

$$
d \sigma(s)=\sum_{N=0}^{\infty} \sigma_{N}(s) \hat{\theta}\left(\alpha^{\prime} s-4 N\right) .
$$

In this paper we have computed two terms $\sigma_{0}(s)$ and $\sigma_{1}(s)$, which are sufficient in order to explore CM energies up to $s=8 M_{s}^{2}$. All remaining terms give vanishing contribution at $s<8 M_{s}^{2}$. It is worth noting that for large $s$ both terms $\sigma_{0}(s)$ and $\sigma_{1}(s)$ go exponentially to zero, due to the factor $2^{-2 \alpha^{\prime} s}$ in (2.17) and (2.18). It is plausible that this will be true for all $\sigma_{N}$, i.e. that each individual term (corresponding to all string contributions of a given mass $M=$ $2 \sqrt{N} M_{s}$ ) is exponentially suppressed at large $s$. The ex- 
plicit form of $\sigma_{0}$ and $\sigma_{1}$ suggests that at large $s$ a generic term should have the form $\sigma_{N}(s) \cong c_{N} \alpha^{\prime} \alpha_{s}^{2}\left(\alpha^{\prime} s\right)^{2 N}\left(\alpha^{\prime} s-\right.$ $4 N)^{2} 2^{-2 \alpha^{\prime} s}$. It would be interesting to see if this is indeed the case.

In conclusion, the cross section for the leading missing transverse momentum process exhibits a typical pattern with peaks and zeros at periodic values of the CM energy, where the widths and the heights of the peaks are given in terms of the string scale $M_{s}$ and $\alpha_{s}$. We provide a quantitative picture of this pattern, taking a basic string model, see Figs. 2 and 3. We hope that the present results may help to look for experimental signals of genuine string-theory effects at high energy colliders.

\section{ACKNOWLEDGMENTS}

The authors acknowledge partial support by the ECRTN network MRTN-CT-2004-005104. D. C. and R. I. also acknowledge partial support by the Italian MIUR program "Teoria dei Campi, Superstringhe e Gravità." J. R. also acknowledges partial support by MCYT FPA 2004-04582-C02-01 and CIRIT GC 2001SGR-00065.

\section{APPENDIX: CROSS SECTIONS IN PRESENCE OF RESONANCES}

The formula (3.1) can be obtained from unitarity, as follows.

Near resonances, we expect the $S$ matrix to have the following form:

$$
S=S_{0}+\frac{R_{1}}{E-E_{r}+i \frac{\Gamma_{1}}{2}}+\frac{R_{2}}{E-E_{r}+i \frac{\Gamma_{2}}{2}}
$$

where we have supposed that there are two resonances at the same energy with two different decay widths.

By unitarity:

$$
S^{\dagger} S=1
$$

whence

$$
\left\{\begin{array}{l}
S_{0}^{\dagger} S_{0}=1 \\
\left(E-E_{r}+i \frac{\Gamma_{i}}{2}\right) R_{i}^{\dagger} S_{0}+\left(E-E_{r}-i \frac{\Gamma_{i}}{2}\right) S_{0}^{\dagger} R_{i}+R_{i}^{\dagger} R_{i}=0 \\
R_{1}^{\dagger} R_{2}+R_{2}^{\dagger} R_{1}=0 .
\end{array}\right.
$$

We can parametrize

$$
R_{i}=-i \Gamma_{i} A_{i} S_{0}
$$

and the system becomes:

$$
\begin{gathered}
S_{0}^{\dagger} S_{0}=1 ; \quad A_{i}^{\dagger}=A_{i} ; \quad A_{i}^{2}=A_{i} ; \\
A_{1}^{\dagger} A_{2}+A_{2}^{\dagger} A_{1}=0 .
\end{gathered}
$$

Therefore we can write

$$
A^{i}=A_{\text {out,in }}^{i}=\sum_{r_{i}} u_{\text {out }}^{\left(r_{i}\right)} u_{\text {in }}^{\left(r_{i}\right) *}
$$

where $r_{i}$ are quantum numbers distinguishing the various resonant states and $u_{s t}^{r}$ are orthonormal vectors.

Using for states |in $\rangle$ and |out $\rangle$ a partial wave basis $\left|j, j^{z},\{n\}\right\rangle$, where $j$ is the total angular momentum, $j^{z}$ is its third component and $\{n\}$ is a collection of the other quantum number characterizing the state, due to angular momentum conservation we can write

$$
u_{j_{i},\{n\}}^{j_{i}^{z}}=\delta_{j_{i}, j_{i n}} \delta_{j_{i}^{z}, j_{i n}^{z}} u_{\{n\}}
$$

The cross section for a process $a \rightarrow b$ is then (see [16], Chap. 3)

$$
\sigma=\frac{16}{s} \frac{\pi}{\mathcal{N}} \sum_{j}(2 j+1) \frac{M_{r}^{2} \Gamma_{j a} \Gamma_{j b}}{\left(s-M_{r}^{2}\right)^{2}+\Gamma_{j}^{2} M_{r}^{2}},
$$

where $\mathcal{N}$ is a numerical factor giving the multiplicity of the initial states and $\Gamma_{j a(b)}=\Gamma_{j} \sum_{n}\left|u_{\{n\}}^{a(b)}\right|^{2}$ is the partial width corresponding to the decays of resonance $|j\rangle$ into states $|a(b)\rangle$.
[1] N. Arkani-Hamed, S. Dimopoulos, and G. R. Dvali, Phys. Rev. D 59, 086004 (1999).

[2] C. D. Hoyle, D. J. Kapner, B. R. Heckel, E. G. Adelberger, J. H. Gundlach, U. Schmidt, and H.E. Swanson, Phys. Rev. D 70, 042004 (2004); C. D. Hoyle, U. Schmidt, B. R. Heckel, E. G. Adelberger, J.H. Gundlach, D. J. Kapner, and H. E. Swanson, Phys. Rev. Lett. 86, 1418 (2001).

[3] I. Antoniadis, N. Arkani-Hamed, S. Dimopoulos, and G. R. Dvali, Phys. Lett. B 436, 257 (1998).

[4] G. F. Giudice, R. Rattazzi, and J.D. Wells, Nucl. Phys. B544, 3 (1999).

[5] E. A. Mirabelli, M. Perelstein, and M. E. Peskin, Phys. 
Rev. Lett. 82, 2236 (1999).

[6] E. Dudas and J. Mourad, Nucl. Phys. B575, 3 (2000).

[7] S. Cullen, M. Perelstein, and M. E. Peskin, Phys. Rev. D 62, 055012 (2000).

[8] J. L. Hewett, Phys. Rev. Lett. 82, 4765 (1999).

[9] M. Bando, T. Kugo, T. Noguchi, and K. Yoshioka, Phys. Rev. Lett. 83, 3601 (1999).

[10] E. Accomando, I. Antoniadis, and K. Benakli, Nucl. Phys. B579, 3 (2000).

[11] H. Murayama and J. D. Wells, Phys. Rev. D 65, 056011 (2002).

[12] M. B. Green and J.H. Schwarz, Nucl. Phys. B198, 441
(1982).

[13] J. H. Schwarz, Phys. Rep. 89, 223 (1982).

[14] A. Hashimoto and I. R. Klebanov, Phys. Lett. B 381, 437 (1996); Nucl. Phys. B Proc. Suppl. 55, 118 (1997).

[15] D. Chialva, R. Iengo, and J. G. Russo, J. High Energy Phys. 01 (2005) 001.

[16] Steven Weinberg, Foundations, The Quantum Theory of Fields Vol. 1 (Cambridge University Press, Cambridge, England, 1995).

[17] A. Litke et al. (ALEPH Collaboration), CERN Report No. CERN-OPEN-99-269, ALEPH 99-051. 